\title{
ANÁLISE COMPARATIVA DE METODOLOGIAS DE AVALIAÇÃO DE PAVIMENTOS ATRAVÉS DO IGG E PCI
}

\author{
Suyanne Alves Vieira ${ }^{1}$, Antonio Adilson Eufrasino de Pinho Júnior ${ }^{2}$, \\ Francisco Heber Lacerda de Oliveira ${ }^{2}$, Marcos FÁbio Porto de Aguiar ${ }^{1}$ \\ ${ }^{1}$ Instituto Federal de Educação, Ciência e Tecnologia do Ceará, Departamento de Construção Civil \\ ${ }^{2}$ Universidade de Fortaleza, Centro Ciências Tecnológicas \\ <suyanneav@hotmail.com><engadilsonpinho@hotmail.com> \\ $<$ heberoliveiracivil@hotmail.com><marcosporto@ifce.edu.br $>$
}

DOI: 10.21439/conexoes.v10i3.799

\begin{abstract}
Resumo. A determinação da condição de superfície de um pavimento asfáltico, bem como a análise dos tipos e causas de defeitos, é de grande importância para que sejam elaborados projetos adequados de conservação ou mesmo de recuperação das vias, de modo que os recursos financeiros empregados em serviços de manutenção de pavimentos sejam aplicados de forma criteriosa. Por este motivo, o presente artigo busca a aplicação e a análise de dois métodos de avaliação superficial de pavimentos flexíveis, o Î́ndice de Gravidade Global (IGG) e o Pavement Condition Index (PCI), com intuito de destacar por meio de uma análise comparativa a importância técnica e prática dos métodos estudados em pavimentos flexíveis localizados no campus da Universidade de Fortaleza - UNIFOR. Apesar da diversidade em relação a quantidades e tipos de defeitos adotados em cada método, na maior parte dos segmentos avaliados pelos dois métodos, o diagnóstico foi próximo, apresentando a classificação Bom e Regular, respectivamente. Porém, os dois procedimentos estão sujeitos a deixar de avaliar defeitos importantes, constatando-se que o método do PCI mostrou-se mais completo e apropriado.
\end{abstract}

Palavras-chaves: Pavimento. Avaliação superficial. Índice de Gravidade Global. Pavement Condition Index

\begin{abstract}
The determination of the condition of the surface of an asphalt pavement, as well as analysis of their faults and causes, is of great importance in that they are developed appropriate conservation projects or even recovery of the tracks, so that the financial resources employed in services maintenance of pavements are applied carefully. For this reason, the present article the application and evaluation of two methods for superficial evaluation of flexible pavements, the Global Severity Index (IGG) and the Pavement Condition Index (PCI), in order to highlight through a comparative analysis technical and practical importance of the methods studied in flexible pavements located on the campus of the University of Fortaleza - UNIFOR. Despite the diversity in relation to quantities and types of defects used in each method, in most segments evaluated by two methods (IGG and PCI), the diagnosis was close, with the classification Good and Regular and, respectively. However, both procedures are subject to fail to assess major defects is verified that the method of PCI was more complete and appropriate.
\end{abstract}

Keywords: Pavement. Superficial evaluation. Global Severity Index. Pavement Condition Index. 


\section{INTRODUÇÃO}

A utilização de métodos de avaliação da condição estrutural e funcional dos pavimentos, registros dos custos e dos serviços executados, além do desempenho dos pavimentos, está diretamente relacionada às obras prioritárias para a manutenção da rede viária. Um dos principais objetivos da utilização de métodos avaliativos e de manutenção preventiva de um pavimento é a obtenção do melhor retorno possível para os recursos investidos, provendo pavimentos seguros, confortáveis e econômicos aos usuários (FERNANDES JÚNIOR; ODA; SÓRIA, 1996). Além disso, tais métodos devem possibilitar a melhoria da condição dos pavimentos e a redução dos custos de manutenção e reabilitação, e dos custos de operação de veículos.

No entanto, tem-se constatado, frequentemente, a degradação precoce dos pavimentos reabilitados e, também dos novos, levando a vidas úteis menores, do que as inicialmente previstas, trazendo prejuízo ao estado e aos usuários. Isso ocorre, em parte, devido a aplicações de projetos inadequados para as vias, projetos que muitas vezes são elaborados sem um levantamento detalhado do pavimento ou da situação em que o terreno se encontra. Em alguns casos ocorre que os métodos de avaliação são utilizados de forma inadequada ou ineficiente na avaliação do pavimento.

Atualmente existem métodos de avaliação de pavimentos asfálticos que podem passar informações nas quais permitiram saber a real condição da superfície do pavimento asfáltico, e com esses resultados seriam utilizadas as melhores maneiras para que seja realizada uma ação preventiva ou mesmo uma restauração na via.

Neste contexto, o objetivo geral deste trabalho é a análise comparativa entre dois métodos de avaliação de pavimentos flexíveis, o Índice de Gravidade Global (IGG) e o Pavement Condition Index (PCI), com aplicação no campus da Universidade de Fortaleza - UNIFOR.

Com a utilização desses métodos será possível determinar a condição de superfície de um pavimento asfáltico por meio da atribuição a indicadores reconhecidos que classificam seu estado geral, confrontar os resultados obtidos, permitindo observar as vantagens e desvantagens de cada um.

\section{SISTEMA DE GERÊNCIA DE PAVIMENTOS}

Os pavimentos rodoviários representam um valioso patrimônio, cuja conservação e restauração oportunas são essenciais para a sua preservação. Qualquer interrupção ou redução na intensidade ou na frequência dos serviços necessários à manutenção desse patrimônio implica em aumentos substanciais nos custos de operação dos veículos e na necessidade de investimentos cada vez mais vultosos para sua recuperação (DNIT, 2011).

O objetivo principal de um Sistema de Gerência de Pavimentos, ainda segundo DNIT (2011) é alcançar a melhor aplicação possível para os recursos públicos disponíveis e oferecer um transporte rodoviário seguro, compatível e econômico.

\subsection{Avaliação Superficial dos Pavimentos}

A avaliação de pavimentos, de acordo com Haas e Hudson (1978), é uma das principais etapas de um Sistema de Gerência de Pavimentos, pois é por meio dela que se pode verificar se o pavimento necessita manutenção, reconstrução, se foi bem construído e se está atendendo às especificações para as quais foi dimensionado em projeto.

O objetivo principal da avaliação superficial, de acordo com Marcon (1996), é identificar os defeitos existentes. A caracterização dos defeitos é obtida por meio de três requisitos principais, sendo eles:

a) Tipo do defeito: identificação do defeito e classificação conforme a sua origem (causa);

b) Severidade: é o estágio atual da evolução do defeito, refletindo-se no estado de degradação da área do pavimento afetado pelo defeito;

c) Dimensão dos defeitos: anotação da extensão ou área do pavimento afetada por cada tipo de defeito; em algumas metodologias é anotada diretamente a densidade de defeitos, representado a estimativa do percentual da área afetada por defeitos.

A avaliação da superfície consiste em aferir as condições funcionais e estruturais dos pavimentos por meio da identificação das patologias ou defeitos presentes na superfície do pavimento. É realizada por meio de procedimentos padronizados de medidas e inspeções.

Existem diversas metodologias desenvolvidas para a execução desta atividade, sendo utilizadas por órgãos rodoviários, urbanos e aeroportuários. A escolha de uma metodologia deve considerar as características e as peculiaridades dos pavimentos existentes na rede viária em estudo, bem como os objetivos a serem alcançados. De acordo com Marcon (1996), a eficiência destas técnicas é variável e depende principalmente do grau de detalhamento e do número de variáveis a levantar que, consequentemente, elevam os custos da avaliação.

DNIT (2003b) sugere dois procedimentos para a avaliação objetiva das condições de superfícies pavimentadas. O primeiro deles é uma avaliação baseada 
num índice que expressa o estado geral de um pavimento em função da incidência de defeitos, o Índice de Gravidade Global - IGG, que caracteriza o grau de degradação superficial, resultante do levantamento dos defeitos da superfície, quantificando-os e classificandoos, incluindo a medição das profundidades dos afundamentos nas trilhas de rodas. O segundo procedimento visa avaliar a condição de superfície de pavimentos por meio de subtrechos homogêneos e é mais voltado para estudos e pesquisas para gerência de pavimentos.

Outro método utilizado, sobretudo nos Estados Unidos, para avaliação das condições de superfície é o Índice de Condição do Pavimento (Pavement Condition Index - PCI), de acordo com o United States of America Corp of Engineers (USACE, 1982). Foi primeiramente desenvolvido para pavimentos de aeroportos, e posteriormente ampliado para rodovias, ruas e estacionamentos, com base na experiência adquirida, na validação de campo e informações dos engenheiros especialistas do USACE. Esse índice se propõe a determinar o estado atual de um pavimento em termos de sua integridade estrutural e nível de serviço. Fornece, ainda, uma referência numérica para as quantidades, os tipos e as severidades dos defeitos identificados no pavimento inspecionado e, por sua vez, indica a condição do pavimento (OLIVEIRA, 2009).

\section{MATERIAIS E MÉTODOS}

Para este estudo de caso, foi escolhida uma via interna que apresenta um grande fluxo de veículos diário e atende a várias áreas do campus da Universidade de Fortaleza - UNIFOR, perfazendo uma distância de aproximadamente 423 metros, que vai desde a entrada pela Avenida Dr. Valmir Ponte até a rotatória do estacionamento do Bloco M.

\subsection{Método do índice de Gravidade Global (IGG)}

Para a execução deste método, foi realizado um levantamento em campo da quantificação dos defeitos superficiais, conforme DNIT (2003b). O IGG foi determinado de forma amostral para algumas estações (ou áreas de trabalho) com área e distanciamento entre elas prefixados da seguinte forma: nas rodovias de pista simples, as estações deverão foram inventariadas a cada $20 \mathrm{~m}$, alternados entre faixas, portanto, em cada faixa a cada 40m; nas rodovias de pista dupla, a cada $20 \mathrm{~m}$, na faixa mais solicitada pelo tráfego, em cada uma das pistas. A superfície de avaliação corresponde a $3 \mathrm{~m}$ antes e 3 $\mathrm{m}$ após cada uma das estacas demarcadas, totalizando em cada estação uma área correspondente a $6 \mathrm{~m}$ de extensão e largura igual a da faixa a ser avaliada (DNIT) 2003b). A Figura 1 mostra um exemplo das estações em pista simples.

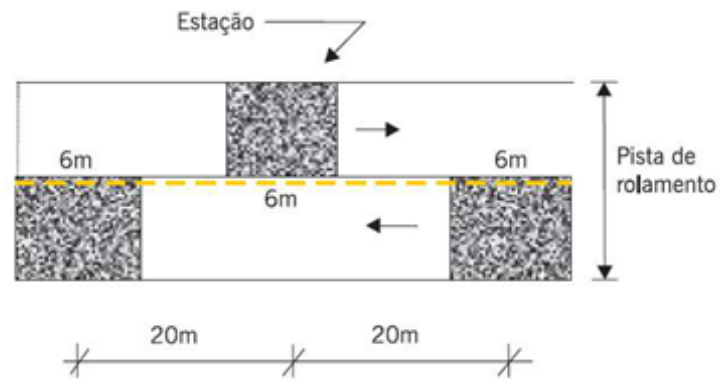

Figura 1: Exemplo de demarcação de áreas para inventário de defeitos.

Fonte: Bernucci et al. 2010).

Na Tabela 1] são apresentados os resultados dos levantamentos realizados em campo nas 20 (vinte) estações de trabalho estudadas.

Para cada uma das ocorrências inventariadas, o Índice de Gravidade Individual foi obtido por meio da fórmula 1, conforme DNIT (2003b):

$$
I G I=f_{r} \times f_{p}
$$

Onde:

$f_{r}=$ frequência relativa;

$f_{p}=$ fator de ponderação.

Para o cálculo do IGI dos afundamentos em trilha de roda, considera-se a média $\mathrm{F}$ dos afundamentos (a norma denomina flechas para os afundamentos nas trilhas de roda) e a média FV das variâncias das flechas de acordo com as fórmulas $2 \mathrm{e} 3$.

$$
\begin{aligned}
& F=\left(\frac{\sum_{i}^{j} F R E+\sum_{i}^{j} F R I}{2}\right) \\
& F V=\left(\frac{F R E_{V}+F R I_{V}}{2}\right)
\end{aligned}
$$

Onde: $F R E=$ flecha na trilha externa em milímetros de cada uma das estações do segmento; $F R I=$ flecha na trilha interna em milímetros de cada uma das estações do segmento; $i$ e $j=$ primeira e última estação do segmento, respectivamente; $F=$ média aritmética da média das flechas na trilha externa e da média das flechas na trilha interna do segmento; $F R E_{v}=$ variância das flechas medidas na trilha externa do segmento, considerando todas as estações de $i$ a $j ; F R I_{v}=$ variância 
ANÁLISE COMPARATIVA DE METODOLOGIAS DE AVALIAÇÃO DE PAVIMENTOS ATRAVÉS DO IGG E PCI

Tabela 1: Planilha empregada para levantamento do estado de superfície segundo IGG.

\begin{tabular}{|c|c|c|c|c|c|c|c|c|c|c|c|c|c|c|c|c|c|c|c|c|c|c|c|}
\hline & & Estação & 0 & 1 & 2 & 3 & 4 & 5 & 6 & 7 & $B$ & 9 & 10 & 11 & 12 & 13 & 14 & 15 & 16 & 17 & 18 & 19 & 20 \\
\hline & & Faixa & D & $E$ & D & $E$ & $\mathrm{D}$ & $E$ & $\mathrm{D}$ & $E$ & D & $E$ & D & $E$ & D & $E$ & D & $E$ & D & D & $E$ & D & $E$ \\
\hline & Conf & gur ação de Terr aplanagem & C & C & $\mathrm{C}$ & C & C & C & $\mathrm{C}$ & C & $5 \mathrm{MC}$ & $5 \mathrm{MC}$ & SMC & $5 \mathrm{MC}$ & SMC & SMC & $5 \mathrm{MC}$ & C & C & C & C & C & C \\
\hline Tipo & OK & Sem defeto & & & $x$ & & & & & & $\mathrm{x}$ & $\mathrm{x}$ & $\mathrm{x}$ & $x$ & & & & & & & & & $x$ \\
\hline 1 & F1 & Fissuras & & & & & & & & & & & & & & & & & $\mathrm{x}$ & & & & \\
\hline $\mathrm{FCl}$ & TTC & Trinces transversais cur tas & & & & & & & & & & & & & $x$ & & & & & & & & \\
\hline & $\mathrm{TTL}$ & Trincas transver sais longas & & & & & & & & & & & & & & & & & & & & & \\
\hline & TLC & Tr incas longitudinais curtas & $\mathrm{x}$ & $\mathrm{x}$ & & & & $\mathrm{x}$ & $\mathrm{x}$ & $\mathrm{x}$ & & & & & & & & & & & & & \\
\hline & TLL & Trincas longitudinais longas & & & & & $\mathrm{x}$ & & & & & & & & & & & & & & & & \\
\hline & TRR & Trincas isoladas retração & $\mathrm{x}$ & & & & & & & & & & & & & & & & & & & & \\
\hline 2 & J & Couro dejacaré & & & & & & & & $x$ & & & & & & & & & & & & & \\
\hline$(\mathrm{FC} I \mathrm{I})$ & $\mathrm{TB}$ & Tr incas em bloco & & & & & & & & & & & & & & & & & & & & & \\
\hline 3 & JE & Couro de jaca é com erosão & & & & & & & & & & & & & & & & & & & & & \\
\hline$(\mathrm{FClII})$ & TBE & Trincas em bloco com erosão & & & & & & & & & & & & & & & & & & & & & \\
\hline 4 & ALP & Afundamento plástico local & & & & & & & & & & & & & & & & & & & & & \\
\hline & ATP & Afundamento plásticotrilha & & & & & & & & & & & & & & & & & & & & & \\
\hline 5 & $\mathrm{OK}$ & Ondulação & & & & & & & & & & & & & & & & & & & & & \\
\hline & $R$ & Panela & & & & $\mathrm{x}$ & & & & & & & & & & & & & & & & & \\
\hline 6 & EX & Exsudação & & & & & & & & & & & & & & $x$ & & & & & & & \\
\hline 7 & $\mathrm{D}$ & Desgaste & & & & $\mathrm{x}$ & $\mathrm{x}$ & & & & & & & & & & $\mathrm{x}$ & $\mathrm{x}$ & & $\mathrm{x}$ & $x$ & $\mathrm{x}$ & \\
\hline 8 & $R$ & Remendo & & & & & & & $\mathrm{x}$ & & & & & & & & & & & & & & \\
\hline & $\mathrm{ALC}$ & Afundamento consolidação local & & & & & & & & & & & & & & & & & & & & & \\
\hline & ATC & Afundamento consolidação triha & & & & & & & & & & & & & & & & & & & & & \\
\hline & EX & Escorr egamento & & & & & & & & & & & & & & & & & & & & & \\
\hline & TRI & Afundamento trilha exter na (mm) & 2 & 5 & 4 & 0 & 1 & 0 & 4 & 0 & 0 & 0 & 2 & 0 & 10 & 6 & 4 & 2 & 1 & 1 & 2 & 1 & 2 \\
\hline & TER & Afundamento trilha interna (mm) & 3 & 3 & 9 & 0 & 1 & 0 & 3 & 0 & 1 & 0 & 0 & 0 & 6 & 8 & 11 & 2 & 2 & 1 & 1 & 0 & 4 \\
\hline
\end{tabular}

das flechas medidas na trilha interna do segmento, considerando todas as estações de $i$ a $j ; F V=$ média da variância das flechas na trilha externa e da variância das flechas na trilha interna do segmento.

A partir do cálculo da média (F) e da variância (FV) das flechas aferidas das TRE e TRI, é que são definidos seus respectivos fatores de ponderação, conforme especificado a seguir:

a) Para valores de média aritmética das medidas das flechas menor ou igual a 30 , o fator de ponderação equivale a 4/3, acima de 30 o IGI e igual a 40.

b) Para valores de média das variâncias das flechas menor ou igual a 50, o fator de ponderação e igual a 1 , acima de 50 o IGI é igual a 50.

O Índice de Gravidade Global (IGG) é calculado pela seguinte expressão 4

$$
I G G=\sum I G I
$$

Em que:

IGG = índice de gravidade global;

IGI = índice de gravidade individual.

Obtendo-se o valor de IGG, é possível estabelecer o conceito do pavimento por meio dos limites apresentados na Tabela 2, conforme DNIT (2003b).
Tabela 2: Condição do Pavimento em função do IGG

\begin{tabular}{cc}
\hline Conceitos & Limites \\
\hline Ótimo & $0<I G G \leq 20$ \\
Bom & $20<I G G \leq 40$ \\
Regular & $40<I G G \leq 80$ \\
Ruim & $80<I G G \leq 160$ \\
Péssimo & $I G G \geq 160$ \\
\hline Fonte: Adaptado de DNIT (2003b).
\end{tabular}

\subsection{Método do Pavement Condition Index ( $\mathrm{PCl})$}

Este método segue USACE (1982) e estabelece que a avaliação da superfície pode ser realizada de forma contínua ou por amostragem. No levantamento realizado, o trecho foi dividido em segmentos com aproximadamente $225 \mathrm{~m}^{2}$. Cada segmento foi inventariado identificando e registrando, manualmente em uma planilha, os tipos, as quantidades e severidade de cada defeito encontrado. Na Tabela 3, são apresentados, resumidamente, os tipos de defeitos considerados e a forma de medição.

O cálculo do PCI foi realizado a partir da equação matemática, como se segue em 5 . 
Tabela 3: Defeitos e forma de medir o PCI

\begin{tabular}{ll|ll}
\hline Defeito & $\begin{array}{l}\text { Forma } \\
\text { de } \\
\text { medição }\end{array}$ & Defeito & $\begin{array}{l}\text { Forma } \\
\text { de } \\
\text { medição }\end{array}$ \\
\hline 1. Couro de Jacaré & área & 11. Remendos & área \\
\hline 2. Exsudação & área & 12. Agregado polido & área \\
\hline 3. Fissuras em blocos & metro & 13. Panelas & unidade \\
\hline 4. Elevações / Recalques & área & 14. Cruzamento ferroviário & área \\
\hline 5. Corrugação & área & 15. Afundamento de trilha de roda & área \\
\hline 6. Afundamento Localizado & área & 16. Escorregamento de massa & área \\
\hline 7. Fissuras de borda & metro & $\begin{array}{l}\text { 17. Fissuras devido ao escorregamento } \\
\text { de massa }\end{array}$ & área \\
\hline 8. Fissuras por reflexão de juntas & metro & 18. Inchamento & área \\
\hline 9. Desnível pavimento / acostamento & metro & 19. Desgaste & área \\
\hline 10. Fissuras longitudinal e transversal & metro & & \\
\hline
\end{tabular}

$$
P C I=100-\sum_{i=1}^{p} \sum_{j=1}^{m_{i}} a\left(T_{i}, S_{j}, D_{i j}\right) F(t, q)
$$

sendo:

$p=$ número total de tipos de defeitos para o pavimento analisado;

$i=$ contador dos tipos de defeitos;

$m_{i}=$ número do nível de severidade para o enésimo tipo de defeito;

$j$ = contador dos níveis de severidade;

$a()=$ valor de dedução;

$F(t, q)=$ função de ajuste para defeitos múltiplos que varia com a soma dos valores de dedução e o número de deduções;

$T_{i}=$ tipos de defeitos;

$S_{j}=$ níveis de severidade;

$D_{i j}=$ densidade.

Os defeitos identificados no campo foram transportados para uma planilha de cálculo de forma a se obter do valor do PCI. Os valores de dedução (a) e a função para ajuste de múltiplos defeitos $(\mathrm{F})$, estão disponíveis graficamente sob forma de curvas nos manuais de USACE (1982).

Como ilustração foi utilizada a Figura 2, que corresponde às trincas couro de jacaré, que é um defeito associado à fadiga do revestimento asfáltico. Após, é necessário realizar o somatório desses valores deduzidos para se obter o Valor Deduzido Total - VDT, que é um valor estabelecido por USACE (1982) para cada tipo de defeito.

Posteriormente, ajusta-se, por meio de um gráfico específico exemplificado na Figura 3, o VDT para um

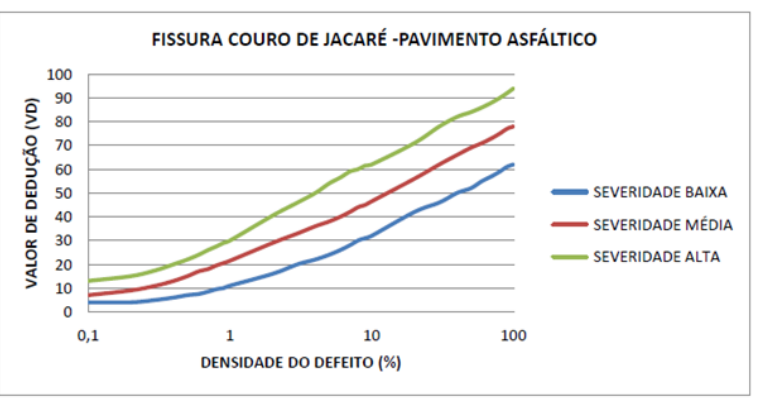

Figura 2: Curvas do valor de dedução para o defeito couro de jacaré. Fonte: adaptado de USACE $(1982)$.

Valor Deduzido Corrigido - VDC, sempre que a quantidade de Valores Deduzidos (q) for superior à classificação cinco.

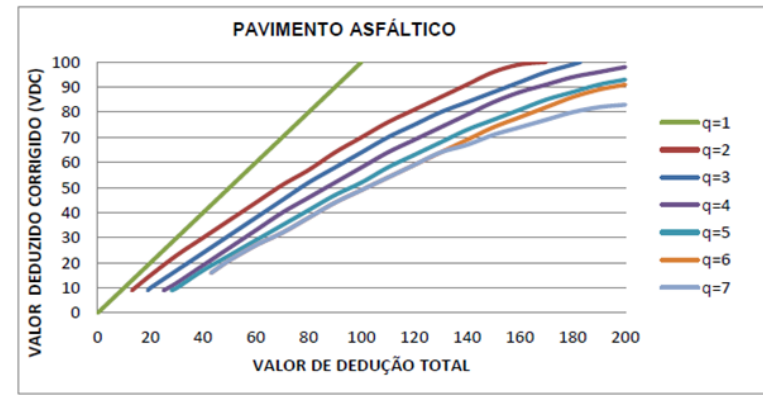

Figura 3: Correção do valor de dedução das curvas de pavimento asfáltico.

Fonte: adaptado de USACE (1982). 
Após obter o VDC, obteve-se o valor do PCI por meio da equação 6 .

$$
P C I=100-V D C
$$

em que:

$P C I$ = índice de condição do pavimento;

$V D C=$ valor deduzido corrigido.

No desenvolvimento do procedimento para determinar o Índice de Condição do Pavimento- PCI (USACE. 1982) foi adotada uma escala a fim de comparar diversos tipos de pavimentos, conforme apresentado na Tabela 4

Tabela 4: Condição do Pavimento em função do PCI.

\begin{tabular}{cc}
\hline PCI & Condição do Pavimento \\
\hline $100-86$ & Excelente \\
$85-71$ & Muito Bom \\
$70-56$ & Bom \\
$55-41$ & Regular \\
$40-26$ & Ruim \\
$25-11$ & Muito Ruim \\
$10-0$ & Péssimo \\
\hline \multicolumn{2}{c}{ Fonte: Adaptado de Shahin (2005). }
\end{tabular}

\section{RESULTADOS E DISCUSSÃO}

De posse dos dados levantados, os cálculos foram realizados para a determinação conceitual de degradação do pavimento.

Na aplicação do método IGG, como a pista estudada tinha variações na sua caixa (largura total da pista), teve que ser segmentado o método de aplicação, sendo a primeira etapa, que vai desde a estação 0 até a estação 12 , uma aplicação em pista dupla, variando a cada 20 metros alternando entre as faixas, e a segunda etapa que vai desde a estação 13 até a estação 20, uma aplicação de pista simples, variando a cada 20 metros.

Para a obtenção dos dados referentes ao método IGG, foi realizada uma análise prévia de forma a subdividir a via em segmentos que possuam as mesmas características ou defeitos. Logo, neste caso, a área avaliada foi subdividida em dois segmentos, e desta forma avaliada como mostra na planilha de cálculo representada pelas Tabelas $5 \mathrm{e} 6$.

As Tabelas 5 e 6 mostram os valores encontrados para IGG do primeiro e segundo segmento que foram de 31,92 e 37,8, respectivamente, e realizando a média dos dois segmentos, encontra-se o valor do IGG de 34,65.

De acordo com DNIT (2003b), o valor encontrado de 34,65 , classifica o pavimento com um conceito de degradação Bom. O valor encontrado é reflexo da recente recuperação do pavimento, haja vista que apresenta poucos defeitos. Outro fator a se ressaltar é a deficiência no método em quantificar os defeitos levantados na pesquisa, fazendo com que a severidade do defeito não seja analisada de forma precisa.

Para o levantamento dos estudos do pavimento por meio do método do PCI foi necessário à marcação da área a ser analisada, como o método estabelece que seja demarcada uma área no total de 225 metros quadrados de forma alternada, e, levando-se em conta que a área total estudada apresenta aproximadamente $1.575,00 \mathrm{~m}^{2}$, totalizando assim um total de quatro segmentos.

Para o cálculo do PCI, pelo método da USACE (1982), uma planilha eletrônica foi elaborada. Nesta planilha foram cadastradas as unidades de defeitos amostradas, os tipos de defeitos e o grau de severidade são identificados de acordo com os apresentados no manual do USACE (1982). Logo em seguida, quantificouse os defeitos apresentando-os por meio da densidade da área afetada.

As Tabelas 7 e 8 mostram os resultados obtidos pelo método que se referem aos levantamentos do segmento 1. O mesmo procedimento foi utilizado para o levantamento e cálculo para a determinação de condição do pavimento para os demais segmentos.

Analisando os resultados, além de indicar um pavimento com poucos defeitos, não foi detectado nesse estudo nenhum defeito de alta severidade em todos os segmentos, o que se caso ocorresse iria acarretar em um pavimento muito deteriorado. Na maioria das áreas os defeitos do pavimento foram considerados de média severidade.

A Tabela 9 apresenta os resultados obtidos neste trabalho discriminados por segmentos avaliados. De acordo com os valores de PCI apresentados na Tabela 9 o trecho estudado constitui-se de segmentos que apresentaram desde condição ruim a boa condição de pavimento, o que reflete na sua funcionalidade para com o usuário desta via.

Observando-se a aplicação dos dois métodos utilizados, pode-se fazer a comparação apresentada na Tabela 10.

Não foram detectados na avaliação do PCI os defeitos panelas, couro de jacaré e remendos. Neste caso, o IGG refletiu melhor a situação existente, pois alguns defeitos de grande importância ficaram fora das 4 amostras selecionadas aleatoriamente no método PCI.

De acordo com Aps, Balbo e Severi (1998), ambos os procedimentos estão sujeitos a deixar de avaliar defeitos importantes, seja devido ao espaçamento cons- 
Tabela 5: Planilha de cálculo do IGI para segmento 1

\begin{tabular}{c|l|l|l|l|l}
\hline Tipo & Natureza do Defeito & $\begin{array}{l}\text { Frequência } \\
\text { Absoluta } \\
\text { Relativa }\end{array}$ & $\begin{array}{l}\text { Frequência } \\
\text { Ponderação }\end{array}$ & $\begin{array}{l}\text { Índice de } \\
\text { Gravidade } \\
\text { Individual }\end{array}$ \\
\hline 1 & $\begin{array}{l}\text { (FCI)F, TTC, TTL, TLC, } \\
\text { TLL, TRR }\end{array}$ & 4 & $31 \%$ & 0,2 & 6,15 \\
\hline 2 & (FCII)J, TB & 0 & $0 \%$ & 0,5 & 0,00 \\
\hline 3 & (FCIII)JE, TBE & 0 & $0 \%$ & 0,8 & 0,00 \\
\hline 4 & ALP, ATP & 0 & $0 \%$ & 0,9 & 0,00 \\
\hline 5 & O, P, E & 1 & $8 \%$ & 1,0 & 7,69 \\
\hline 6 & $\mathrm{E}$ & 0 & $0 \%$ & 0,5 & 0,00 \\
\hline 7 & $\mathrm{D}$ & 2 & $15 \%$ & 0,3 & 4,62 \\
\hline 8 & $\mathrm{R}$ & 1 & $8 \%$ & 0,6 & 4,62 \\
\hline 9 & $\mathrm{~F}=(\mathrm{TRI}+\mathrm{TER}) / 2$ & $\mathrm{TRI}=2,15$ & TER $=2,00$ & $\mathrm{~F}=2,07692$ & 0,52 \\
\hline 10 & $\mathrm{FV}=(\mathrm{TRIv}+\mathrm{TREv}) / 2$ & $\mathrm{TRIv}=8,81$ & TREv $=7,83$ & $\mathrm{FV}=8,32$ & 8,32 \\
\hline$I G I=(F \times 1 / 4)$ quando, $F \leq 30$ & $I G I=F V$ quando, $F V \leq 50$ & & \\
\hline \multicolumn{2}{|l|}{$I G I=40$ quando, $F>30$} & $I G I=560$ quando, $F V>50$ & IGG $=$ & 31,92 \\
\hline
\end{tabular}

Tabela 6: Planilha de cálculo do IGI para segmento 2

\begin{tabular}{|c|c|c|c|c|c|}
\hline Tipo & Natureza do Defeito & $\begin{array}{l}\text { Frequência } \\
\text { Absoluta }\end{array}$ & $\begin{array}{l}\text { Frequência } \\
\text { relativa }\end{array}$ & $\begin{array}{l}\text { Fator de } \\
\text { Ponderação }\end{array}$ & $\begin{array}{l}\text { Índice de } \\
\text { Gravidade } \\
\text { Individual }\end{array}$ \\
\hline 1 & (FCI)F, TTC, TTL, TLC, TLL, TRR & 1 & $13 \%$ & 0,2 & 2,50 \\
\hline 2 & (FCII)J, TB & 0 & $0 \%$ & 0,5 & 0,00 \\
\hline 3 & (FCIII)JE, TBE & 0 & $0 \%$ & 0,8 & 0,00 \\
\hline 4 & ALP, ATP & 0 & $0 \%$ & 0,9 & 0,00 \\
\hline 5 & $\mathrm{O}, \mathrm{P}, \mathrm{E}$ & 0 & $0 \%$ & 1,0 & 0,00 \\
\hline 6 & $\mathrm{E}$ & 1 & $13 \%$ & 0,5 & 6,25 \\
\hline 7 & $\mathrm{D}$ & 5 & $63 \%$ & 0,3 & 18,75 \\
\hline 8 & $\mathrm{R}$ & 0 & $0 \%$ & 0,6 & 0,00 \\
\hline 9 & $\mathrm{~F}=(\mathrm{TRI}+\mathrm{TER}) / 2$ & TRI $=2,38$ & TER $=3,63$ & $\mathrm{~F}=3$ & 0,75 \\
\hline 10 & $\mathrm{FV}=(\mathrm{TRIv}+\mathrm{TREv}) / 2$ & TRIV $=3,13$ & $\mathrm{TREv}=15,63$ & $\mathrm{FV}=9,13$ & 9,13 \\
\hline & & & & IGG $=$ & 37,38 \\
\hline
\end{tabular}


Tabela 7: Planilha para cálculo do Segmento 1.

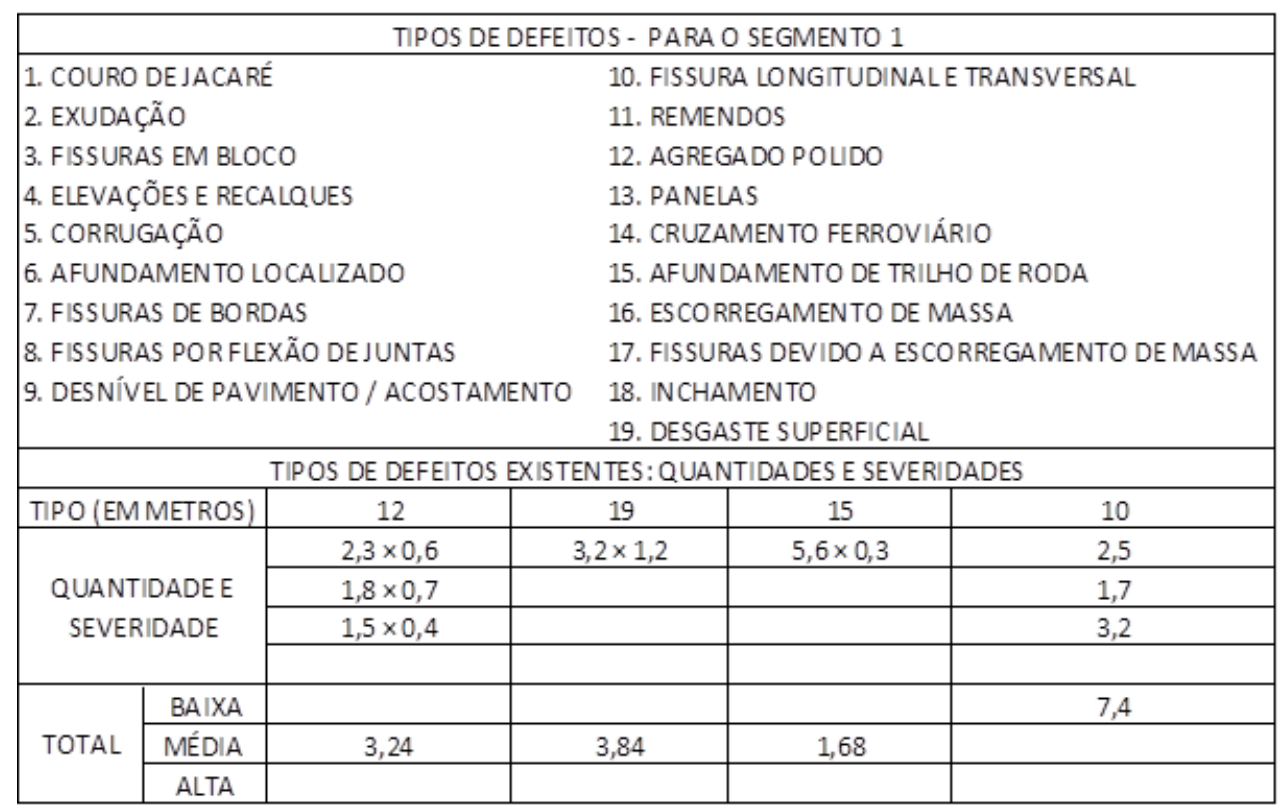

Tabela 8: Planilha para cálculo do Segmento 1.

\begin{tabular}{|c|c|c|c|c|c|c|}
\hline \multicolumn{7}{|c|}{ CÁLCULO DO PCI - PARA O SEGMENTO 1} \\
\hline CÓDIGO DO DEFEITO & TIPO DE DEFEITO & SEVERIDADE & QUANTIDADE & DENSIDADE & VD & $\mathrm{q}$ \\
\hline \multirow{3}{*}{10} & \multirow{3}{*}{$\begin{array}{c}\text { FISSURA LONGITUDINAL } \\
\text { ETRANSVESAL }\end{array}$} & ALTA & 0,00 & 0,00 & 0,00 & 0 \\
\hline & & MÉDIA & 0,00 & 0,00 & $0, \infty 0$ & 0 \\
\hline & & BAIXA & 7,4 & 3,29 & 17,8 & 1 \\
\hline \multirow{3}{*}{12} & \multirow{3}{*}{ AGREGADO POLIDO } & ALTA & $0, \infty 0$ & 0,00 & 0,00 & 0 \\
\hline & & MÉDIA & 3,24 & 1,44 & 23,20 & 1 \\
\hline & & BAIXA & 0,00 & $0, \infty$ & 0,00 & 0 \\
\hline \multirow{3}{*}{15} & \multirow{3}{*}{$\begin{array}{l}\text { AFUNDAMENTO DE } \\
\text { TRILHO DE RODA }\end{array}$} & ALTA & 0,00 & 0,00 & 0,00 & 0 \\
\hline & & MÉDIA & 1,68 & 0,75 & 18,30 & 1 \\
\hline & & BAIXA & 0,00 & 0,00 & 0,00 & 0 \\
\hline \multirow{3}{*}{19} & \multirow{3}{*}{ DESGASTE SUPERFICIAL } & ALTA & $0, \infty 0$ & 0,00 & $0, \infty 0$ & 0 \\
\hline & & MÉDIA & 3,84 & 1,71 & 27,55 & 1 \\
\hline & & BAIXA & $0, \infty 0$ & $0, \infty 0$ & 0,00 & 0 \\
\hline & & & & TOTAL = & 86,85 & 4 \\
\hline & $q=$ & 4 & & & & \\
\hline & valor da dedução total $=$ & 86,85 & & & & \\
\hline valor de & dedução corrigido $($ VDC $)=$ & 52,47 & & & & \\
\hline & $\mathrm{PCl}=$ & 47,53 & & & & \\
\hline & CLASSIFICAÇÃO $=$ & REGULAR & & & & \\
\hline
\end{tabular}


Tabela 9: Classificação do trecho estudado de acordo com o método PCI.

\begin{tabular}{ccc}
\hline & PCI & Conceito \\
\hline Segmento 1 & 47,53 & Regular \\
Segmento 2 & 61,29 & Bom \\
Segmento 3 & 40,45 & Ruim \\
Segmento 4 & 67,66 & Bom \\
Média & 54,23 & Regular \\
\hline
\end{tabular}

Tabela 10: Análise comparativa entre os métodos IGG e PCI.

\begin{tabular}{|c|c|c|}
\hline Método & IGG & PCI \\
\hline Valor & 34,65 & 54,23 \\
\hline Conceito & Bom & Regular \\
\hline $\begin{array}{l}\text { Número de } \\
\text { amostras }\end{array}$ & 21 & 4 \\
\hline $\begin{array}{l}\text { Área amos- } \\
\text { tral }\end{array}$ & $20,64 m^{2}$ & $225,0 \mathrm{~m}^{2}$ \\
\hline $\begin{array}{l}\text { Área total } \\
\text { avaliada }\end{array}$ & $408,0 \mathrm{~m}^{2}$ & $900 \mathrm{~m}^{2}$ \\
\hline $\begin{array}{l}\text { Defeitos } \\
\text { encontrados }\end{array}$ & $\begin{array}{l}\text { Trincas transvesais } \\
\text { curtas (FC1), trincas } \\
\text { longitudinais cur- } \\
\text { tas (FC1), trincas } \\
\text { longitudinais longas } \\
\text { (FC1), trincas iso- } \\
\text { ladas (FC1), trincas } \\
\text { couro de jacaré } \\
\text { (FC2), afundamen- } \\
\text { tos de trilha de roda, } \\
\text { panelas, desgaste, } \\
\text { remendo. }\end{array}$ & $\begin{array}{l}\text { Agregado po- } \\
\text { lido, desgaste, } \\
\text { afundamentos } \\
\text { de trilha de } \\
\text { roda, fissuras } \\
\text { longitudinal e } \\
\text { transversal. }\end{array}$ \\
\hline
\end{tabular}

Conex. Ci. e Tecnol. Fortaleza/CE, v. 10, n. 3, p. 20 - 30, nov. 2016 tante do método IGG ou a aleatoriedade da escolha de amostras método do PCI.

\section{ANÁLISE CRÍTICA DAS METODOLOGIAS ABORDADAS}

Este trabalho teve por objetivo principal analisar tecnicamente dois métodos de avaliação do pavimento, no caso o método do Índice de Gravidade Global - IGG (DNIT, 2003b) e o método do Pavement Condition Index - PCI (USACE, 1982), bem como sua importância para avaliar e conceituar o atual estágio em que se encontra o pavimento, utilizando como base as patologias existentes.

De um modo geral, foi explanado sobre a composição dos pavimentos, patologias de pavimentos e a importância que se faz presente as avaliações dos pavimentos para uma adequada solução e/ou manutenção periódica que deva vir a ser executada.

Em relação às tipologias de degradações do revestimento asfáltico, observou-se uma grande diversidade de defeitos adotados para cada método. Os métodos divergem na quantidade e nos tipos de defeitos, além das diferenças de nomenclatura para os defeitos com a mesma descrição de morfologia e gênese.

O método do Índice de Gravidade Global - IGG não define níveis de severidade para os defeitos, porém alguns tipos são diferenciados por características que denotam a severidade do defeito, como no caso das trincas tipo couro de jacaré, distintos pela presença ou não de erosão nos bordos.

Verificou-se também que alguns defeitos considerados nos métodos não são significativos nos pavimentos urbanos. No método do IGG são registradas separadamente as trincas que ocorrem nas bordas do pavimento. No meio urbano, em geral, os pavimentos são dotados de contenção lateral, localizada entre a pista de rolamento e o passeio publico, reduzindo significativamente a incidência deste tipo de defeito. No método do $\mathrm{Pa}$ vement Condition Index (PCI), as elevações e recalque são considerados um tipo único de defeito, porém estas desagregações possuem morfologia e gêneses distintas, além de necessitarem de tipos de serviços diferenciados.

Entre os dois modelos empregados para a determinação do Índice da Condição do Pavimento, constatouse que o método do PCI mostra-se mais completo e apropriado, pois no cálculo são considerados os tipos de defeitos, os níveis de severidade, a área afetada, além da quantidade de defeitos existentes na seção avaliada. Porém os dois modelos apresentam defeitos em seus levantamentos, pois foram detectados alguns defeitos que não tinham como ser avaliados por meio de ambos os 
métodos, como, por exemplo, a presença de um galho de arvora dentro da estrutura do pavimento, possivelmente, foi inserida durante a execução do serviço.

Constatou-se com os estudos que o pavimento analisado apresenta uma classificação ruim em uma de suas partes, o que não era esperado, pois o pavimento é de recente execução. Essas imperfeições fazem com que o conforto e a qualidade diminuam bastante, haja vista que o pavimento que acabou de ser construído ou reformado deveria esta em excelente condição de uso.

\section{CONSIDERAÇÕES FINAIS}

Houve certa dificuldade em ressaltar alguns defeitos encontrados nos pavimentos, pois os métodos não conseguem abordar $100 \%$ dos defeitos diagnosticados nos levantamentos realizados em campo. Mesmo assim, ficou claro que a existência desses defeitos afeta a qualidade da via, por mais que não esteja expressa nos métodos utilizados.

Confrontando os dois modelos foi possível verificar que o método do Pavement Condition Index - PCI apresenta uma melhor composição para a análise do pavimento, isso ocorre devido a forma como é abordado e o detalhamento exigido em seus cálculos, fazendo que o seu resultado seja mais preciso do que o do método do Índice de Gravidade Global - IGG.

Na prática, a aplicação dos dois modelos é viável, porém o método do IGG é mais fácil de utilizar devido à simplicidade na hora de executar o seu levantamento em campo, sendo essa sua única vantagem em relação do método do PCI que não se trata de um procedimento trivial, necessitando de um maior aparato para que o levantamento seja feito de forma satisfatória, além de ter uma relativa demora na coleta de dados em campo.

Assim, o uso do IGG é mais indicado quando se necessita de uma avaliação imediata e em grandes extensões, funcionando como uma ferramenta para a alimentação de dados de um sistema de gerência a nível de rede. Já a aplicação do método PCI é mais adequado para trechos menores, quando se há a necessidade de o projetista de quantificar o volume de tapa-buracos, as extensões de remendos localizados, pequenas áreas que necessitem de fresagem, dentre outros.

Conclui-se que os procedimentos para levantamento e os defeitos elencados são adequados à avaliação da condição superficial dos pavimentos urbanos e viável aplicação em nível de rede viária. É necessário, no entanto, aprimorar aplicação dos métodos com a inserção dos defeitos que não são computados para a análise mais precisa do método.

A via analisada foi escolhida devido ao seu grande fluxo de veículos diários e por ser uma via de grande im- portância para a locomoção do trânsito dentro do campus. A metodologia estudada deveria ser aplicada a todos as vias da UNIFOR, pois com uma análise bem efetuada, os pavimentos da universidade teriam os dados necessários para que fossem efetuadas as manutenções e/ou reformas que possam vir a ser necessárias.

\section{REFERÊNCIAS}

APS, M.; BALBO, J. T.; SEVERI, A. A. Avaliação superficial de pavimentos asfálticos em vias urbanas utilizando o método do pci. In: ABPV. REUNIÃO ANUAL DE PAVIMENTAÇÃO,. São Paulo, 1998. v. 31.

BERNUCCI, L. B.; MOTTA, L. M. G.; CERATTI, J. A. P.; SOARES, J. B. Pavimentação asfáltica: Formação básica para engenheiros. Rio de Janeiro: PETROBRAS, ABEDA, 2010.

DNIT. Norma 005/2003: Defeitos nos Pavimentos Flexíveis e Semi-rígidos Terminologia. Rio de Janeiro: DNIT, Departamento Nacional de Infra-estrutura de Transportes, 2003a.

. Norma PRO 006/2003: Avaliação Objetiva da Superfície de Pavimentos Flexíveis e Semi-Rígidos. Rio de Janeiro: DNIT, Departamento Nacional de Infra-estrutura de Transportes, 2003b.

Manual de Gerência de Pavimentos - IPR

745/2011. Rio de Janeiro: DNIT, Departamento Nacional de Infra-estrutura de Transportes, 2011.

FERNANDES JÚNIOR, J. L.; ODA, S.; SÓRIA, M. H. A. Caracterização e levantamento das estradas municipais para fins de gerências de vias. In: ABPV. REUNIÃO DE PAVIMENTAÇÃO URBANA - RPU. São José dos Campos, 1996. v. 7.

HAAS, R.; HUDSON, W. Pavement Management Systems. New York: Mcgraw-Hill, 1978.

MARCON, A. F. Contribuições ao Desenvolvimento de um Sistema de Gerência de Pavimentos para a Malha Rodoviária Estadual de Santa Catarina. Tese (Doutorado) — Instituto Tecnológico da Aeronáutica, São José dos Campos, 1996.

OLIVEIRA, F. H. L. Proposição de Estratégias de Manutenção de Pavimentos Aeroportuários Baseadas na Macrotextura e no Atrito: Estudo de caso do Aeroporto Internacional de Fortaleza. Dissertação (Mestrado) — Programa de Mestrado em Engenharia de Transportes, Universidade Federal do Ceará, Fortaleza, 2009. 
SHAHIN, M. Y. Pavement Management for Airports, Roads and Parking Lots. second. New York: Chapman \& Hall, 2005.

USACE. Pavement Maintenance Management.

Technical Manual - TM 5-623. Washington: USACE,

United State Army Corps of Engineers, Headquarters,

Department of the Army, 1982. 